\title{
Two permutation classes related to the Bubble Sort operator
}

\author{
Marilena Barnabei Flavio Bonetti Matteo Silimbani \\ Dipartimento di Matematica \\ Università di Bologna \\ Italy \\ \{marilena.barnabei,flavio.bonetti,matteo.silimbani4\}@unibo.it
}

Submitted: Feb 7, 2012; Accepted: Aug 17, 2012; Published: Aug 30, 2012

Mathematics Subject Classifications: 05A05, 05A15, 68P10

\begin{abstract}
We introduce the Dual Bubble Sort operator $\hat{B}$ (a sorting algorithm such that, if $\sigma=\alpha 1 \beta$ is a permutation, then $\hat{B}(\sigma)=1 \alpha \hat{B}(\beta))$ and consider the set of permutations sorted by the composition $\hat{B} B$, where $B$ is the classical Bubble Sort operator. We show that this set is a permutation class and we determine the generating function of the descent and fixed point distributions over this class. Afterwards, we characterize the same distributions over the set of permutations that are sorted by both $\hat{B}^{2}$ and $B^{2}$.
\end{abstract}

Keywords: permutation class, sorting algorithm, permutation statistic.

\section{Introduction}

A permutation $\sigma$ is said to contain a permutation $\tau$ if there exists a subsequence of $\sigma$ that has the same relative order as $\tau$, and in this case $\tau$ is said to be a pattern of $\sigma$. Otherwise, $\sigma$ is said to avoid the pattern $\tau$.

A class of permutations is a downset in the permutation pattern order defined above. Every class $C$ can be defined by its basis $B$, namely, the set of minimal permutations that are not contained in it, and we write $C=A v(B)$. We denote by $A v_{n}(B)$ the set $A v(B) \cap S_{n}$.

Permutation classes arise naturally when one studies the behaviour of some well-known sorting algorithms. More precisely, those algorithms that have the property that, if they are able to sort a permutation $\sigma$, then they sort any subpermutation of $\sigma$.

The first example was given by D.E.Knuth, who proved in [4] that the set of permutations sorted by a single application of the Stack Sort operator is the class $A v(231)$. The action 
of the Stack Sort operator has been extensively studied after the seminal paper by J.West [9].

A further example is the Bubble Sort operator $B$ that can be recursively described as follows: $B(\epsilon)=\epsilon$, where $\epsilon$ is the empty permutation, and, if $\sigma=\alpha n \beta$ is a non-empty permutation, and $n$ its maximal value, then

$$
B(\sigma)=B(\alpha) \beta n \text {. }
$$

The Bubble Sort operator has the nice property (not shared by the Stack Sort operator, see [9]) that the set of permutations that are sorted by any power of $B$ form a class, as proved in [2]. Further properties of the Bubble Sort algorithm have been recently studied in [1].

An apparently new trivial variation $\hat{B}$ of the operator $B$ can be defined as follows: $\hat{B}(\epsilon)=\epsilon$ and, if $\sigma=\alpha 1 \beta$ is a non-empty permutation, then

$$
\hat{B}(\sigma)=1 \alpha \hat{B}(\beta) \text {. }
$$

We call $\hat{B}$ the Dual Bubble Sort operator.

We observe that the Dual Bubble Sort operator $\hat{B}$ is related to the operator $B$ by

$$
\hat{B}=\rho B \rho,
$$

where $\rho$ is the usual reverse-complement operator.

Note that the composition $C=\hat{B} B$ is another classical sorting operator introduced by Knuth in [5], namely, the Cocktail Shaker Sort operator, also known as the Bidirectional Sort operator.

In the first part of this paper we consider the set of permutations that are sorted by a single run of the Cocktail (Shaker) Sort operator $C$. We show that this set is the class $A v(3412,3421,4312,4321)$, and enumerate the elements in $A v_{n}(3412,3421,4312,4321)$. Furthermore, we study the distributions of descents and fixed points over this class. In particular, we show that fixed point free permutations in the class are enumerated by Pell numbers.

The second part of the paper is devoted to the study of the set of permutations that are sorted by both $B^{2}$ and $\hat{B}^{2}$, namely, permutations in $B^{-2}(i d) \cap \hat{B}^{-2}(i d)$. This set turns out to be the class $A v(S)$, where $S$ is the set of patterns of length 4 of type either $4 x x x$ or $x x x 1$. The set $B^{-2}(i d) \cap \hat{B}^{-2}(i d)$ can be also characterized as the set of permutations $\sigma$ satisfying $|\sigma(i)-i| \leqslant 2$ for all $i$. The more general problem of studying the set $T_{d, n}$ of permutations of length $n$ which satisfy $|\sigma(i)-i| \leqslant d$ (whose motivation comes from coding theory) was first discussed by R.Lagrange in [6], next by D.H.Lehmer in [7] and lately by T.Kløve in [3].

We show that the set $T_{2, n}$ has the peculiar property that, for $n \geqslant 5$, it contains only two connected permutations. A permutation $\sigma \in S_{n}$ is connected if it does not have a prefix $\sigma^{\prime}$ of length $k<n$ that is a permutation of the symbols $1,2, \ldots, k$. This property allows us to determine the joint distribution of descents and fixed points over $T_{2, d}$. In fact, the property above smoothes the way to the study of the distribution of several other permutation statistics over $T_{2, n}$. As an example, we deduce the generating function of the joint distribution of descents and occurrences of the pattern 321 over $T_{2, n}$. 


\section{The Cocktail Sort algorithm}

The purpose of this section is to study the set of permutations sorted by a single run of the Cocktail Sort algorithm $C$. First of all we show that this set is indeed a class, whose basis is finite:

Theorem 1. A permutation $\sigma \in C^{-1}(i d)$ if and only if $\sigma$ avoids the patterns 3412, 3421, 4312 , and 4321.

Proof. It is well known ([1], see also [2]) that the Bubble Sort algorithm is such that

$$
B(\sigma)=i d \Longleftrightarrow \sigma \in A v(321,231)
$$

and, hence,

$$
\hat{B}(\sigma)=i d \Longleftrightarrow \sigma \in A v(321,312)
$$

This implies that

$$
C(\sigma)=i d \Longleftrightarrow B(\sigma) \in A v(321,312) \Longleftrightarrow \sigma \in B^{-1}(A v(321,312)) .
$$

Proposition 8 in [1] states that if $\pi \in S_{n-1}(n \geqslant 4), \pi=n-1 \pi^{\prime}$, then

$$
B^{-1}(A v(\pi))=A v\left(n-1 n \pi^{\prime}, n n-1 \pi^{\prime}\right) .
$$

This result implies that

$$
C(\sigma)=i d \Longleftrightarrow \sigma \in A v(3412,3421,4312,4321) .
$$

The preceding theorem yields immediately the following result that will be useful in the rest of this section:

Proposition 2. Let $\sigma \in A v(3412,3421,4312,4321)$. If the maximal symbol $n$ appears in $\sigma$ at position $i \leqslant n-2$, then the symbol $n-1$ must be placed either at position $n$ or $n-1$.

Denote by $c_{n}$ the cardinality of the set $A v_{n}(3412,3421,4312,4321)$. We determine the generating function of the sequence $c_{n}$ by considering first the connected permutations in $A v_{n}(3412,3421,4312,4321)$.

Proposition 3. Denote by $c c_{n}$ the number of connected permutations in $A v_{n}(3412,3421,4312,4321)$. Then, for $n \geqslant 2$,

$$
c c_{n}=3^{n-2}
$$

Proof. We observe that 21 is the only connected permutation in $A v_{2}(3412,3421,4312,4321)$. Let now $n$ be an integer, $n \geqslant 3$. Every connected permutation $\sigma$ in $A v_{n-1}(3412,3421,4312,4321)$ yields exactly 3 different connected permutations of length $n$ in the same class either by: 
i) replacing the symbol $n-1$ by $n$, and inserting $n-1$ at the last position, or

ii) replacing the symbol $n-1$ by $n$, and inserting $n-1$ at the second last position, or

iii) inserting the symbol $n$ at the second last position.

On the other hand, it is easily seen that the same operations applied to a non-connected permutation yield longer non-connected permutations.

Now, by Proposition 2, all connected permutations in $A v_{n}(3412,3421,4312,4321)$ are obtained in this way.

Theorem 4. The generating function of the sequence $c_{n}$ is

$$
\Gamma(x)=\sum_{n \geqslant 0} c_{n} x^{n}=\frac{1-3 x}{1-4 x+2 x^{2}} .
$$

Proof. By Proposition 3, the generating function of the sequence $c c_{n}$ is the following:

$$
C \Gamma(x)=\sum_{n \geqslant 1} c c_{n} x^{n}=x+\frac{x^{2}}{1-3 x} .
$$

Recall that every permutation $\sigma$ can be decomposed into a non-empty connected prefix $\alpha$ and an arbitrary suffix $\beta$ and that $\sigma$ belongs to the class $A v(3412,3421,4312,4321)$ if and only if both $\alpha$ and $\beta$ avoid the same patterns. This implies that

$$
\Gamma(x)=\frac{1}{1-C \Gamma(x)} .
$$

We observe that the sequence $c_{n}$ appears (shifted by one term) as seq. A006012 in [8], even though the current interpretation is not present.

We now turn our attention to the study of the descent distribution on $A v(3412,3421,4312,4321)$. We recall that a permutation $\sigma$ has a descent at position $i$ whenever $\sigma(i)>\sigma(i+1)$.

We first study the case of connected permutations, which can be divided into 3 types, according to the generation rule described in the proof of Proposition 3. More precisely, we say that a connected permutation $\alpha$ in $A v_{n}(3412,3421,4312,4321)$ is:

- of type 0 if $\alpha$ is obtained from a permutation $\sigma \in A v_{n-1}(3412,3421,4312,4321)$ by applying the operation i);

- of type 1 if $\alpha$ is obtained from a permutation $\sigma \in A v_{n-1}(3412,3421,4312,4321)$ by applying the operation ii); 
- of type 2 if $\alpha$ is obtained from a permutation $\sigma \in A v_{n-1}(3412,3421,4312,4321)$ by applying the operation iii).

Let $\sigma \in A v_{n-1}(3412,3421,4312,4321)$ be a permutation with $k$ descents. Denote by $\sigma^{(0)}$, $\sigma^{(1)}$, and $\sigma^{(2)}$ the 3 permutations of length $n$ generated by $\sigma$. For example, if $\sigma=31524$, then

$$
\sigma^{(0)}=316245 \quad \sigma^{(1)}=316254 \quad \sigma^{(2)}=315264 .
$$

Moreover, we have:

- if $\sigma$ is of type 0 , then $\sigma^{(0)}$ has $k$ descents, while $\sigma^{(1)}$ and $\sigma^{(2)}$ have $k+1$ descents;

- if $\sigma$ is of type 1 , then also $\sigma^{(0)}, \sigma^{(1)}$, and $\sigma^{(2)}$ have $k$ descents;

- if $\sigma$ is of type 2 then $\sigma^{(1)}$ has $k+1$ descents, while $\sigma^{(0)}$ and $\sigma^{(2)}$ have $k$ descents.

Let $c c_{n, k}$ denote the number of non-empty connected elements in $A v_{n}(3412,3421,4312,4321)$ with $k$ descents. Then, we have:

Proposition 5. The bivariate generating function of the sequence $c c_{n, k}$ is

$$
C \Psi(x, y)=\sum_{\substack{n \geqslant 1 \\ k \geqslant 0}} c c_{n, k} x^{n} y^{k}=\frac{x-3 x^{2}+x^{2} y+3 x^{3}-4 x^{3} y+x^{3} y^{2}-x^{4}+2 x^{4} y-x^{4} y^{2}}{1-3 x+3 x^{2}-3 x^{2} y-x^{3}+2 x^{3} y-x^{3} y^{2}}
$$

Proof. Denote by $a_{n, k}^{(i)}, i=0,1,2$, the number of connected permutations in $A v_{n}(3412,3421,4312,4321)$ of type $i$ with $k$ descents, hence, $c c_{n, k}=a_{n, k}^{(0)}+a_{n, k}^{(1)}+a_{n, k}^{(2)}$. The following recurrences can be easily deduced from previous considerations:

$$
\begin{gathered}
a_{n, k}^{(0)}=a_{n-1, k}^{(0)}+a_{n-1, k}^{(1)}+a_{n-1, k}^{(2)}=c c_{n-1, k} \\
a_{n, k}^{(1)}=a_{n-1, k-1}^{(0)}+a_{n-1, k}^{(1)}+a_{n-1, k-1}^{(2)} \\
a_{n, k}^{(2)}=a_{n-1, k-1}^{(0)}+a_{n-1, k}^{(1)}+a_{n-1, k}^{(2)}
\end{gathered}
$$

These three identities yield the following relations, for $n \geqslant 4$ :

$$
\begin{gathered}
c c_{n, k}=2 c c_{n-1, k}+c c_{n-1, k-1}-c c_{n-2, k}+c c_{n-2, k-1}+a_{n-1, k}^{(1)}-a_{n-1, k-1}^{(1)}, \\
a_{n, k}^{(1)}=c c_{n-1, k-1}-a_{n-1, k-1}^{(1)}+a_{n-1, k}^{(1)} .
\end{gathered}
$$

Let now

$$
C \Psi^{(1)}(x, y)=\sum_{\substack{n \geqslant 1 \\ k \geqslant 0}} a_{n, k}^{(1)} x^{n} y^{k}
$$

be the generating function of the sequence $a_{n, k}^{(1)}$. Identities (1) and (2) yield

$$
C \Psi(x, y)=x-2 x^{2}+x^{3}-x^{3} y
$$




$$
\begin{gathered}
+\left(2 x+x y-x^{2}+x^{2} y\right) C \Psi(x, y)+(x-x y) C \Psi^{(1)}(x, y), \\
C \Psi^{(1)}(x, y)=\frac{(C \Psi(x, y)-x) x y}{1-x+x y}
\end{gathered}
$$

where the correction terms of $x$-degree less than 4 are due to the fact that Identities (1) and (2) hold for $n \geqslant 4$. Combining Identities (3) and (4) we get the assertion.

We can exploit the above result to deduce the descent distribution over the set $A v(3412,3421,4312,4321)$, using the same arguments as in the proof of Proposition 4:

Theorem 6. The bivariate generating function of the sequence $c_{n, k}$ is

$$
\begin{gathered}
\Psi(x, y)=\sum_{n, k \geqslant 0} c_{n, k} x^{n} y^{k}=\frac{1}{1-C \Psi(x, y)}= \\
=\frac{1-3 x+3 x^{2}-3 x^{2} y-x^{3}+2 x^{3} y-x^{3} y^{2}}{1-4 x+6 x^{2}-4 x^{2} y-4 x^{3}+6 x^{3} y-2 x^{3} y^{2}+x^{4}-2 x^{4} y+x^{4} y^{2}} .
\end{gathered}
$$

The first values of the integers $c_{n, k}$ are shown in the following table:

\begin{tabular}{l|lllllll}
$n / k$ & 0 & 1 & 2 & 3 & 4 & 5 & 6 \\
\hline 1 & 1 & & & & & & \\
2 & 1 & 1 & & & & & \\
3 & 1 & 4 & 1 & & & & \\
4 & 1 & 10 & 9 & & & & \\
5 & 1 & 20 & 41 & 6 & & & \\
6 & 1 & 35 & 133 & 61 & 2 & & \\
7 & 1 & 56 & 350 & 336 & 49 & & \\
8 & 1 & 84 & 798 & 1336 & 465 & 20 & \\
9 & 1 & 120 & 1638 & 4300 & 2789 & 380 & 4
\end{tabular}

Now we want to study the distribution of fixed points on the set $A v(3412,3421,4312,4321)$, namely, the sequence $f_{n, h}$ of the number of permutations in $A v_{n}(3412,3421,4312,4321)$ with $h$ fixed points. First of all, we have the following result:

Proposition 7. The number of fixed point-free elements in $A v_{n}(3412,3421,4312,4321)$, $n \geqslant 1$, is the $(n-1)$-th Pell number $P_{n-1}$, namely,

$$
f_{n, 0}=P_{n-1} .
$$

Proof. It is well known that the Pell numbers (see seq. A000129 in [8]) satisfy the following recurrence:

$$
P_{0}=0, \quad P_{1}=1, \quad P_{n}=2 P_{n-1}+P_{n-2}(n>1) .
$$

On the other hand, it is easily checked that

$$
f_{1,0}=0, \quad f_{2,0}=1 .
$$


Let $\sigma$ be a fixed point free permutation in $A v_{h}(3412,3421,4312,4321)$. Then, we can construct three longer fixed point free permutations in $A v(3412,3421,4312,4321)$ either by

a. adding the symbols $h+2$ and $h+1$ at the two last positions in this order, or

b. inserting the symbol $h+1$ at the second last position, or

c. replacing the symbol $h$ with $h+1$ and inserting $h$ at the end of the permutation.

It is an immediate consequence of Proposition 2 that every fixed point free permutation in $A v_{n}(3412,3421,4312,4321), n \geqslant 1$, is obtained exactly once in this way. This yields:

$$
f_{n, 0}=2 f_{n-1,0}+f_{n-2,0},
$$

as wished.

The integers $f_{n, h}, h>0$, can be determined making use of the following result:

Proposition 8. Let $\sigma$ be a permutation in $A v(3412,3421,4312,4321)$, and $\sigma^{\prime}$ be the permutation obtained from $\sigma$ by adding a fixed point $p$ and increasing by one all the symbols $e \geqslant p$. Then $\sigma^{\prime} \in A v(3412,3421,4312,4321)$.

Proof. Suppose that $\sigma^{\prime}$ contains the pattern $c d a b$, with $l=\max (a, b)<c, d$. Since $\sigma$ avoids $c d a b$, the fixed point $p$ must appear in this pattern. Suppose that $p=c$ and let $j$ be the position of $a$ in $\sigma^{\prime}$. In this case, the $j-3$ positions of $\sigma$ preceding $j$ and not containing $c$ and $d$ can be occupied only by symbols less then $l$. We have $l-2$ of these symbols different from $a$ and $b$. Since $l<c<j$, we have $l-2 \leqslant j-4$, hence we get a contradiction.

The last result implies that every permutation $\sigma \in A v_{n}(3412,3421,4312,4321)$ with $h$ fixed points can be uniquely obtained from a fixed point free permutation in $A v_{n-h}(3412,3421,4312,4321)$. Hence:

Theorem 9. We have:

$$
f_{n, h}=\left(\begin{array}{l}
n \\
h
\end{array}\right) P_{n-h-1}
$$

The first values of the integers $f_{n, k}$ are shown in the following table:

\begin{tabular}{l|llllllllll}
$n / k$ & 0 & 1 & 2 & 3 & 4 & 5 & 6 & 7 & 8 & 9 \\
\hline 2 & 1 & 0 & 1 & & & & & & & \\
3 & 2 & 3 & 0 & 1 & & & & & & \\
4 & 5 & 8 & 6 & 0 & 1 & & & & & \\
5 & 12 & 25 & 20 & 10 & 0 & 1 & & & & \\
6 & 29 & 72 & 75 & 40 & 15 & 0 & 1 & & & \\
7 & 70 & 203 & 252 & 175 & 70 & 21 & 0 & 1 & & \\
8 & 169 & 560 & 812 & 672 & 350 & 112 & 28 & 0 & 1 & \\
9 & 408 & 1521 & 2520 & 2436 & 1512 & 630 & 168 & 36 & 0 & 1
\end{tabular}




\section{Permutations sorted by both $B^{2}$ and $\hat{B}^{2}$}

It has been proved in [2] that a permutation $\sigma$ is sorted by $k$ iterations of the Bubble Sort operator whenever $i-\sigma(i) \leqslant k$ for every $i$. It is straightforward to verify that a permutation has the property above if and only if it avoids every pattern of length $k+2$ of type $x x x \ldots x 1$.

Recall that

$$
\hat{B}=\rho B \rho,
$$

where $\rho$ is the usual reverse-complement operator. This implies immediately that a permutation $\sigma$ is sorted by $k$ iterations of $\hat{B}$ whenever $\sigma(i)-i \leqslant k$ for every $i$, or equivalently, if and only if $\sigma$ avoids every pattern of length $k+2$ of type $(k+2) x x x \ldots x$. In particular, we have:

Theorem 10. A permutation $\sigma$ belongs to $B^{-2}(i d) \cap \hat{B}^{-2}(i d)$ if and only if

- $|\sigma(i)-i| \leqslant 2$ for every $i$, or equivalently

- $\sigma$ belongs to $A v(S)$, where $S$ is the set of patterns of length 4 of type either $4 x x x$ or $x x x 1$.

From now on, we will denote by $T_{2}$ the set $B^{-2}(i d) \cap \hat{B}^{-2}(i d)$. Obviously, $T_{2} \cap S_{n}=T_{2, n}$. The sequence of the cardinalities of the sets $T_{2, n}$ appears in [8] as seq. A002524.

The set $T_{2, n}$ has the peculiar property that, for $n \geqslant 5$, it contains only two connected permutations. More precisely, we have:

Proposition 11. The only connected elements in $T_{2}$ are:

a. the permutations 1, 21, 321, 231, 312 and 3412;

b. the permutation $\pi_{n} \in S_{n}, n \geqslant 4$, defined as follows:

- if $n=2 s$

$$
\begin{cases}\pi_{2 s}(1)=2 & \\ \pi_{2 s}(2 s)=2 s-1 & \\ \pi_{2 s}(2 h)=2 h+2 & 1 \leqslant h \leqslant s-1 \\ \pi_{2 s}(2 h+1)=2 h-1 & 1 \leqslant h \leqslant s-1\end{cases}
$$

- if $n=2 s+1$

$$
\begin{cases}\pi_{2 s+1}(1)=2 & \\ \pi_{2 s+1}(2 s)=2 s+1 & \\ \pi_{2 s+1}(2 h)=2 h+2 & 1 \leqslant h \leqslant s-1 \\ \pi_{2 s+1}(2 h+1)=2 h-1 & 1 \leqslant h \leqslant s\end{cases}
$$

c. the permutation $\tau_{n}:=\pi_{n}^{-1}, n \geqslant 4$. 
Proof. It is obvious that the listed permutations are connected elements in $T_{2}$ and that, for $n \leqslant 4$, these are the only ones. Let now $\sigma$ be a connected permutation in $T_{2, n}, n \geqslant 5$. It is evident that $\sigma(1)$ is either 2 or 3 . In the first case, $\sigma(2) \neq 1$, otherwise $\sigma$ is not connected. Furthermore, $\sigma(2) \neq 3$, otherwise $\sigma(3)$ would be forced to be equal to 1 , contradicting the connectedness condition. Iterating these arguments, we deduce that, if $\sigma(1)=2$, then $\sigma=\pi_{n}$.

The case $\sigma(1)=3$ can be treated similarly, getting $\sigma=\tau_{n}$.

For example, we have

$$
\begin{aligned}
& \pi_{6}=241635 \\
& \tau_{6}=315264,
\end{aligned}
$$

and

$$
\begin{aligned}
& \pi_{7}=2416375 \\
& \tau_{7}=3152746 .
\end{aligned}
$$

We examine the generating function

$$
A(x, y, z)=\sum_{n} \sum_{\sigma \in T_{2, n}} x^{n} y^{\operatorname{des}(\sigma)} z^{f i x(\sigma)}=\sum_{n, d, r} t_{n, d, r} x^{n} y^{d} z^{r}
$$

of the joint distribution of descents and fixed points on the set $T_{2}$. Here the integer $t_{n, d, r}$ denotes the number of permutations in $T_{2, n}$ with $d$ descents and $r$ fixed points. To this aim, we first study the generating function of the same distribution on the set $C T_{2}=\bigcup_{n \geqslant 0} C T_{2, n}$ of non-empty connected permutations in $T_{2}$.

Denote by $c t_{n, d, r}$ the number of elements in $C T_{2, n}$ with $d$ descents and $r$ fixed points. As an immediate consequence of Proposition 11, we have:

Proposition 12. The only non-zero coefficients ct $_{n, d, r}$ are the following:

a. $c t_{1,0,1}=c t_{2,1,0}=c t_{3,2,1}=c t_{4,2,0}=1 ;$

b. $c t_{3,1,0}=c t_{4,1,0}=2$;

c. if $n \geqslant 5, n=2 s, c t_{2 s, s-1,0}=c t_{2 s, s, 0}=1$;

d. if $n \geqslant 5, n=2 s+1, c t_{2 s+1, s, 0}=2$.

Denote by

$$
C A(x, y, z)=\sum_{n} \sum_{\sigma \in C T_{2, n}} x^{n} y^{\operatorname{des}(\sigma)} z^{f i x(\sigma)}=\sum_{n, d, r} c t_{n, d, r} x^{n} y^{d} z^{r} .
$$

Proposition 12 allows us to find an expression for $C A(x, y, z)$. In fact, we have:

$$
\begin{gathered}
C A(x, y, z)=x z+x^{2} y+2 x^{3} y+x^{3} y^{2} z+2 x^{4} y+x^{4} y^{2}+\sum_{s \geqslant 3}\left(x^{2 s} y^{s-1}+x^{2 s} y^{s}\right)+\sum_{s \geqslant 2} 2 x^{2 s+1} y^{s}= \\
x z+x^{2} y+2 x^{3} y+x^{3} y^{2} z+2 x^{4} y+x^{4} y^{2}+\frac{x^{5} y^{2}(2+x+x y)}{1-x^{2} y} .
\end{gathered}
$$

This gives the following result: 


\section{Proposition 13.}

$$
C A(x, y, z)=\frac{x z+x^{2} y+2 x^{3} y-x^{3} y z+x^{3} y^{2} z+2 x^{4} y-x^{5} y^{3} z-x^{6} y^{2}}{1-x^{2} y} .
$$

Every $\sigma \in T_{2}$ can be represented as the juxtaposition of a connected non-empty prefix $\bar{\sigma}$ and a suffix $\sigma^{\prime}$. Needless to say, both $\bar{\sigma}$ and $\sigma^{\prime}$ (up to renormalization) belong to $T_{2}$, $\operatorname{des}(\sigma)=\operatorname{des}(\bar{\sigma})+\operatorname{des}\left(\sigma^{\prime}\right)$, and $f i x(\sigma)=f i x(\bar{\sigma})+f i x\left(\sigma^{\prime}\right)$. This allows us to determine an expression for the generating function $A(x, y, z)$ :

Theorem 14. We have:

$$
\begin{gathered}
A(x, y, z)=\frac{1}{1-C A(x, y, z)}= \\
=\frac{1-x^{2} y}{1-x z-2 x^{2} y-2 x^{3} y+x^{3} y z-x^{3} y^{2} z-2 x^{4} y+x^{5} y^{3} z+x^{6} y^{2}} .
\end{gathered}
$$

As a final observation, we point out that every connected permutation in $T_{2}$ except 321 avoids the pattern 321. Hence, the joint distribution of descents and occurrences of the pattern 321 can be easily obtained by similar arguments:

$$
\begin{gathered}
B(x, y, t)=\sum_{n} \sum_{\sigma \in T_{2, n}} x^{n} y^{\operatorname{des}(\sigma)} t^{o c c_{321}(\sigma)}= \\
=\frac{1-x^{2} y}{1-x-2 x^{2} y-x^{3} y-x^{3} y^{2} t-2 x^{4} y+x^{5} y^{3} t+x^{6} y^{2}}
\end{gathered}
$$

where $\operatorname{occ}_{321}(\sigma)$ denotes the number of occurrences of the pattern 321 in $\sigma$.

\section{Acknowledgments}

We thank the referee for many precious suggestions.

\section{References}

[1] M.H.Albert, M.D.Atkinson, M.Bouvel, A.Claesson, M.Dukes. On the inverse image of pattern classes under bubble sort. Journal of Combinatorics 2 (2):231-243, 2011.

[2] F.Chung, A.Claesson, M.Dukes, R.Graham. Descent polynomials for permutations with bounded drop size. European J. Combin. 31:1853-1867, 2010.

[3] T.Kløve. Generating functions for the number of permutations with limited displacement. Electron. J. Combin. 16:\#R104 2009.

[4] D.E.Knuth. The art of computer programming, Vol. 1: "Fundamental algorithms". Addison-Wesley, Reading MA, 1975. 
[5] D.E.Knuth. The art of computer programming, Vol. 3: "Sorting and searching".. Addison-Wesley, Reading MA, 1973.

[6] R.Lagrange. Quelques résultats dans la métrique des permutations. Annales Scientifiques de l'École Normale Supérieure 79:199-241, 1962.

[7] D.H.Lehmer. Permutations with strongly restricted displacements. Combinatorial theory and its applications, II (Proc. Colloq., Balatonfured, 1969), pp. 755-770. North-Holland, Amsterdam, 1970.

[8] N.J.A.Sloane. The On-Line Encyclopedia of Integer Sequences. http://www . research.att.com/ njas/sequences/.

[9] J.West. Sorting twice through a stack. Theoretical Comput. Sci. 117:303-313, 1993. 